\title{
Discharge of a granular silo under mechanical vibrations
}

\author{
Arthur Pascot ${ }^{1, *}$, Ghita Marouazi ${ }^{1}$, and Sébastien Kiesgen De Richter ${ }^{1, * *}$ \\ ${ }^{1}$ LEMTA (UMR 7563 CNRS), 2 Avenue de la Forêt de Haye, 54505 Vandoeuvre-lès-Nancy Cedex, France.
}

\begin{abstract}
In this paper, we study the flow rate of model granular material in a silo under the influence of mechanical vibrations. Experimental measurements and discrete element simulations (DEM) are performed in a quasi-2D silo. The influence on the flow rate of the opening size and the vibration applied on the entire silo is studied. Two distinct regimes are evidenced, governed by the Froude number $F r$ and the relative frequency $\Omega$. In the first regime, a decreased flow rate is observed when increasing the vibration intensity. This behavior is explained by the presence of reorganizations induced by the vibration, leading to a more homogeneous but also slower flow. In the second regime, an increased flow rate is evidenced when increasing the vibration intensity. We find this behavior comes from the intermittent nature of the flow, where the flow rate is directly controlled by the propagation of shock waves all along the silo.
\end{abstract}

\section{Introduction}

Understanding the behavior and flow properties (blocking, localization, aging) of granular materials is still a major issue in fluid mechanics and rheology, mainly due to the multi-scale aspect of the flow. Their comprehension is however necessary to optimize granular flows, such as silo discharge.

The flow rate of a granular silo has been extensively studied, starting with Beverloo et al. [1]. The authors have shown that the flow rate mostly depends on the opening size and particle properties and proposed an empirical law linking them. More recent studies have evidenced limits to this law, in particular when the opening size tends to the particle diameter [2-4] and for more complex flows $[5,6]$. Different corrections have been proposed to predict the flow rate in those cases, generally by taking into account the decrease of volume fraction at the opening due to collisions [2-4]. However, those laws do not take into account the clogging of the silo due to the formation of arches at the opening [7, 8]. To avoid these arches, a solution is to apply mechanical vibrations to the silo $[9,10]$. Indeed, vibrations can suppress the yield stress and change the rheology of granular flows [11-13].

In this paper, we performed experiments and simulations to explore the influence of vibrations on the flow rate. In Sec. 2, the experimental setup and numerical model used are presented. In Sec. 3, the influence of the opening size and vibrations parameters on the flow rate is presented. Lastly, two regimes are analyzed and discussed separately while a complete empirical law is proposed.

\footnotetext{
*e-mail: arthur.pascot@univ-lorraine.fr

**e-mail: sebastien.kiesgen@univ-lorraine.fr

A video is available at https://doi.org/10.48448/2dxh-y852
}

\section{Methods}

The experimental setup used (see figure 1a) is composed of a rectangular transparent silo $(H=300 \mathrm{~mm}, L=100$ $\mathrm{mm}$ ) mounted on a vertical shaker. The opening size $D$ of the silo can be freely changed to fit different configurations. Similarly to our previous setup [14] and other studies $[3,8,10]$, the silo is quasi-2D with a depth $W=1.5$ $\mathrm{mm}$ which allows the flow of only one layer of particles. This configuration allows a $2 \mathrm{D}$ flow which can be recorded with a camera and prevents wall effects in the other directions. To avoid static electricity during the discharge, the silo is made of anti-static PMMA and an anti-static coating is also applied regularly.

The particles used are non-cohesive spherical glass beads (Silibeads type $S$ ) with a diameter $d=1.1 \pm 0.1$ $\mathrm{mm}$. These particles are collected in a container using a hopper on an articulated arm. The container is placed on a dynamic scale which measures the mass flow rate ${ }^{1}$. The shaker applies vertical and sinusoidal vibrations, whose amplitude $A$ and frequency $f$ can be finely tuned. Vibrations can be applied up to a maximum relative acceleration $\Gamma=A \omega / g=11$, where $g$ the gravitational acceleration and $\omega=2 \pi f$ the pulsation. This is the main change compared to the previous setup [14], which could only reach $\Gamma=3.3$.

Discrete element simulations (DEM) are also performed using the open-source software LIGGGHTS [15]. The geometry and vibrations are the same as those used in experiment. A snapshot of the simulated silo is presented in figure $1 \mathrm{~b}$. During the discharge, contacts between particles and with the walls are resolved using a "soft sphere" approach (Young modulus $E<1 \times 10^{9}$ ) and the "Hertz" contact model developed by Di-Renzo et al. [16]. The

\footnotetext{
${ }^{1}$ It should also be noted that to ensure the repeatability of the results, each measure of flow rate in experiment and simulation is performed three times.
} 

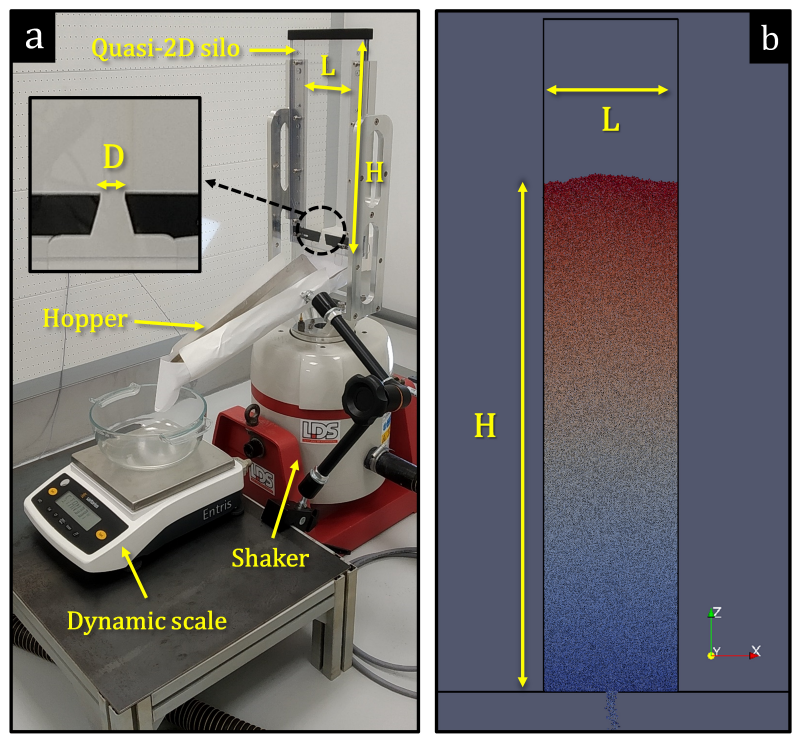

Figure 1. (a) Side view of the experimental setup and close-up of the opening ( $D=10 \mathrm{~mm}$ on the picture). (b) Snapshot of the simulated system with the same geometry as the experiment. Particles are colored in order of insertion, from blue to red.

Table 1. Particle properties used in the simulation: Young modulus $E$ and coefficients of Poisson $v$, restitution $e$ and friction $\mu$. The subscripts $p p$ and $p w$ indicate particle-particle and particle-wall contacts, respectively.

\begin{tabular}{c|cccc}
\hline Parameter & $E$ & $v$ & $e_{p p} / e_{p w}$ & $\mu_{p p} / \mu_{p w}$ \\
\hline Value & $1 \times 10^{9}$ & 0.22 & $0.97 / 0.83$ & $0.4 / 0.15$ \\
\hline
\end{tabular}

physical properties used are based on the literature and calibrated on the experiment without vibration (see table 1).

\section{Results}

The evolution of the normalized flow rate $Q / Q_{0}\left(Q_{0}\right.$ being the flow rate without vibration) with the vibration amplitude $A=0-2800 \mu \mathrm{m}$ was measured for a opening size $D \sim 10 \mathrm{~mm}$ and a frequency $f=30 \mathrm{~Hz}$, as presented in figure 2. The experimental and numerical results are in good agreement ${ }^{2}$ and two distinct regimes are observed:

- A first regime at low amplitude, where the flow rate decreases with the vibrations.

- A second regime at high amplitude, where the flow rate increases with the vibrations.

In order to understand the origin of these regimes, we analyze and discuss them separately.

\subsection{First regime}

The evolution of the normalized flow rate $Q / Q_{0}$ with the amplitude $A$ was measured in experiment, as presented in figure $3 \mathrm{a}$. The measurements were performed for four

\footnotetext{
${ }^{2}$ The small discrepancies observed are mostly due to the different opening sizes.
}

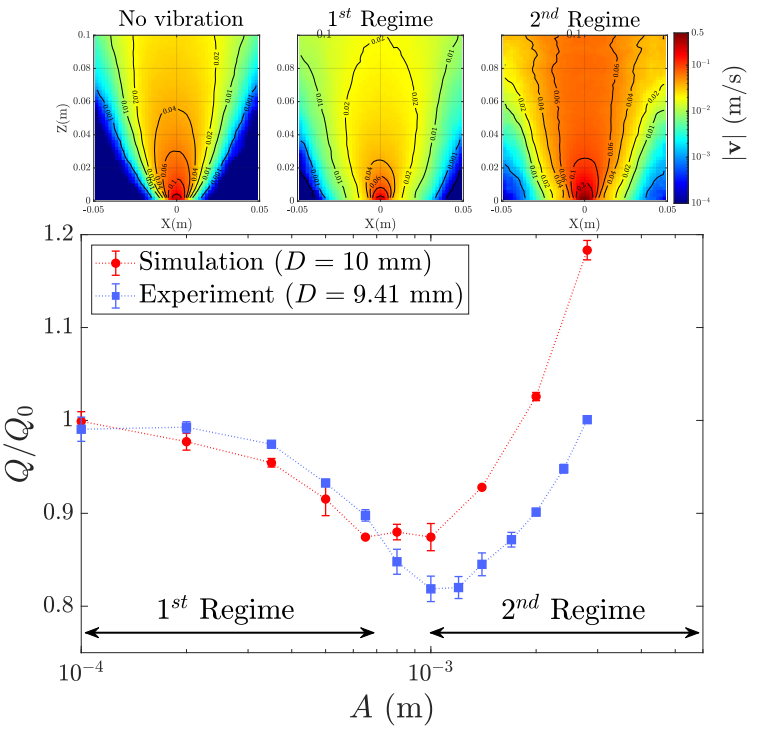

Figure 2. Normalized flow rate $Q / Q_{0}$ as a function of the vibration amplitude $A$ in experiment and simulation for a frequency $f=30 \mathrm{~Hz}$ and an opening size $D \sim 10 \mathrm{~mm}$. Two different regimes are observed for the flow rate depending on the amplitude. To illustrate, typical profiles of mean relative velocity $|v|$ near the silo opening are presented for those regimes and without vibration in simulation (Note: the relative velocities are always measured in the referential of the silo: $v=v_{\text {absolute }}-v_{\text {silo }}$ ).

opening sizes ${ }^{3} D=4.99-21.53 \mathrm{~mm}$ at a given frequency $f=30 \mathrm{~Hz}$ and four frequencies $f=30-75 \mathrm{~Hz}$ at a given opening $D=9.41 \mathrm{~mm}$. In each case, we remain in the first regime $(\Gamma \lesssim 3)$. A coupling with the opening size $D$ and the frequency $f$ is observed for the decrease of flow rate with the amplitude: the normalized flow rate decreases faster for the low frequencies and small opening sizes. Those results are in agreement with the study of Wassgren et al. [17] for a 3D silo and our previous experimental results [14].

The evolution of the normalized flow rate can be scaled with the Froude number $F r=A \omega / \sqrt{g D}$ introduced by Wassgren et al. [17], as shown in figure 3b. The experimental and numerical data are well fitted (solid line in figure $3 \mathrm{~b}$ ) to a simple exponential law based on a Weibull model [18], such as:

$$
\frac{Q}{Q_{0}}=1-a_{1} \exp \left(-b_{1} / F r\right)
$$

with $a_{1}$ and $b_{1}$ two fitting constants. This decrease of flow rate with the Froude number can result from a greater number of contacts between particles due to the vibrations. Indeed, vibrations set into motion previously dead zones on the side of the silo (see the velocity profiles in figure 2), leading to an increase of the competitiveness at the opening as described by Pastor et al.[19]. In addition, vibrations also increase the particle paths in the flow channel and thus the number of contacts.

\footnotetext{
${ }^{3}$ Those limits on the opening size ensure that a constant flow rate can be observed for at least $2 \mathrm{~s}$ without vibration and to avoid wall effects. Note that clogging can still be observed for $D=4.99 \mathrm{~mm}$, in particular without vibration.
} 


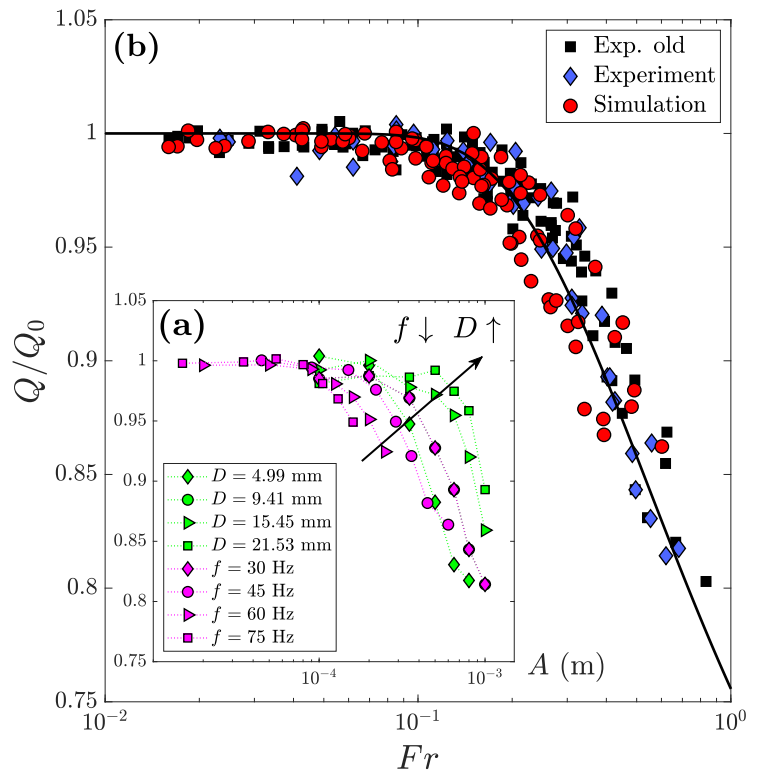

Figure 3. (a) Dimensionless flow rate $Q / Q_{0}$ as a function of the amplitude $A$ for the different opening sizes $D=4.99-21.53 \mathrm{~mm}$ ( $f=30 \mathrm{~Hz}$, in green) and frequencies $f=30-75 \mathrm{~Hz}(D=$ $9.41 \mathrm{~mm}$, in magenta). (b) Dimensionless flow rate $Q / Q_{0}$ as a function of the Froude number $F r$ for all cases in experiment and simulation (including previous results [14], noted "Exp. old"). The data are fitted to equation 1 , with $a_{1}=0.420$ and $b_{1}=0.542$ $\left(R^{2}=908\right)$.

The scaling proposed here is quite similar to the one proposed in our previous study[14] but better captures the influence of the frequency of vibration in the second regime where shock waves propagate.

\subsection{Second regime}

In order to understand the origin of the second regime, the flow at the opening was recorded in experiment and simulation for $D \sim 10 \mathrm{~mm}, f=30 \mathrm{~Hz}$ and $A=2800$ $\mu \mathrm{m}$. During the discharge, the flow rate oscillates between important bursts of particles and periods with a near-zero flow rate (figure 4a). This dynamic is periodic with the vibration velocity and is due to the propagation of waves all along the silo (see figure $4 \mathrm{~b}-\mathrm{c}$ ). Those waves, created by the vibrations and the gravity, lead to periodic shocks and compressions near the opening, as observed for vibrated granular beds $[20,21]$. This behavior is similar to piston engine, which ejects the particles at the opening in the form of periodic bursts.

Since the mechanisms behind each regime are different, we assume that their contributions can be separated, such as:

$$
\frac{Q}{Q_{0}}=1+\mathcal{F}_{1}+\mathcal{F}_{2} \quad \text { with } \quad \mathcal{F}_{1}=-a_{1} \exp \left(-b_{1} / F r\right)
$$

where $\mathcal{F}_{1}$ is the contribution of the first regime described previously and $\mathcal{F}_{2}$ the contribution of the second regime. The evolution of the normalized flow rate $\mathcal{F}_{2}$ with the Froude $\mathrm{Fr}$ was measured for the previous cases and a
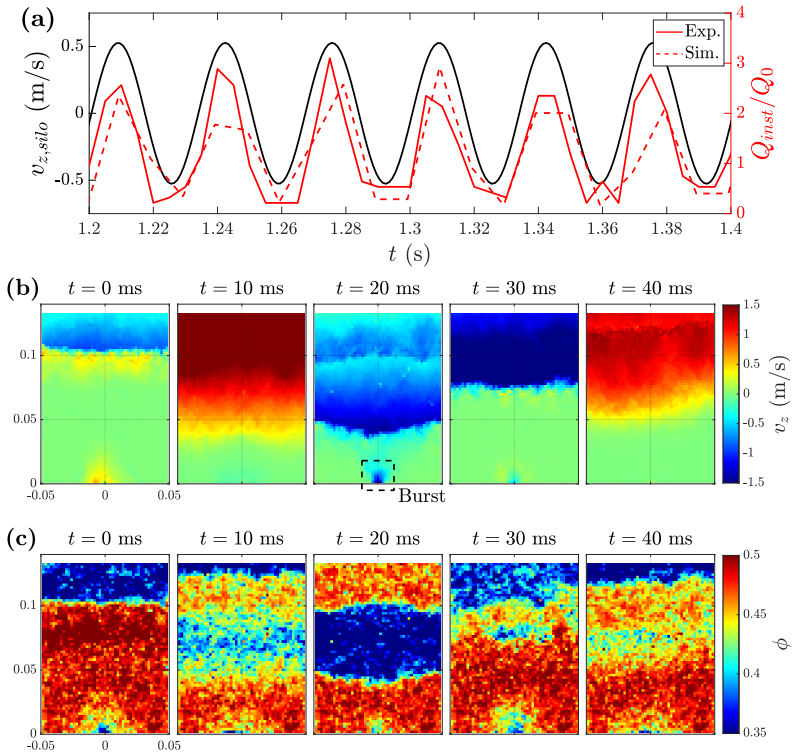

Figure 4. (a) "Instantaneous" relative flow rate $Q_{\text {inst }} / Q_{0}$ (measured every $10 \mathrm{~ms}$ ) and $v_{z, \text { silo }}$ during the discharge in experiment and simulation. A periodic dynamic is observed in phase with $v_{z, \text { silo }}$ : burst of flow rate when the silo goes up (positive peaks of $v_{z, \text { silo }}$ ) and nearly no flow when it goes down (negative peaks of $v_{z, s i l o}$ ). This dynamic comes from the propagation of compression waves in the silo as shown by the profiles of (b) relative vertical velocity $v_{z}$ and (c) volume fraction $\phi$ (in simulation, $A=8000$ $\mu \mathrm{m})$.

larger range of acceleration $\Gamma=0-11$, as shown in figure 5a. An exponential increase with a supplementary contribution of the frequency $f$ (in addition to the Froude number) is observed, in agreement with previous numerical results [14]. To rationalize our data, we introduce the relative frequency $\Omega=\omega / \sqrt{g / d}$. This quantity compares the fall time of a particle to the period of the vibrations and is used in the compaction of granular bed under vibrations [22]. The best scaling for the normalized flow rate $\mathcal{F}_{2}$ is obtained for $F r \Omega^{1 / 2}$, as presented in figure 5b. By analogy to an activation process and the first regime, we propose the following equation for the second regime:

$$
\mathcal{F}_{2}=a_{2} \exp \left(\frac{-b_{2}}{F r \Omega^{1 / 2}}\right)
$$

with $a_{2}$ and $b_{2}$ two fitting constants. The experimental and numerical data are well fitted to this equation with $a_{2}=$ 2.18 and $b_{2}=3.86$.

\section{Conclusion}

In this paper, we studied the discharge of model granular materials inside a quasi-2D silo under vibrations. The evolution of the flow rate with the vibration amplitude and frequency and the opening size was investigated using experiment and discrete element simulation (DEM). Two distinct regimes were evidenced in each case:

- The first regime, where the flow rate decreases when increasing the vibrations. This regime is caused by 


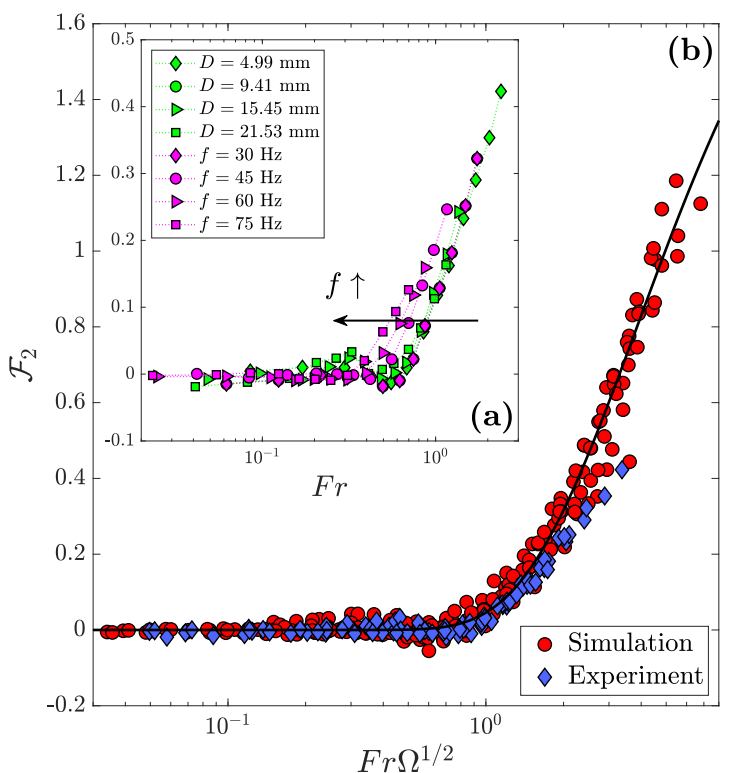

Figure 5. (a) Dimensionless flow rate $\mathcal{F}_{2}$ as function of the Froude number $F r$ for the different opening sizes $D=4.99-$ $21.53 \mathrm{~mm}(f=30 \mathrm{~Hz}$, in green) and frequencies $f=30-75$ $\mathrm{Hz}\left(D=9.41 \mathrm{~mm}\right.$, in magenta). (b) Dimensionless flow rate $\mathcal{F}_{2}$ as function of $F r \Omega^{1 / 2}$ for all cases in experiment and simulation. The data are fitted to equation 3 , with $a_{2}=2.18$ and $b_{2}=3.86$ $\left(R^{2}=0.985\right)$.

an higher competitiveness at the opening and longer paths in the flow channel. This decrease scales with the Froude number $F r$.

- The second regime, where the flow rate increases when increasing the vibrations. This regime is due to the propagation of shock waves in the silo, creating periodic compressions and bursts of particles at the opening. This increase scales with the Froude number $F r$ and the relative frequency $\Omega$.

More complex cases could be studied in a future work, in particular the influence of cohesion on the discharge and the case of a 3D geometry.

\section{Acknowledgements}

This study is conducted in the framework of the "PowderReg" project, funded by the European programme Interreg VA GR within the priority axis 4 "Strengthen the competitiveness and the attractiveness of the Grande Région / Großregion".

\section{References}

[1] W.A. Beverloo, H.A. Leniger, J. Van de Velde, Chem. Eng. Sci 15, 260 (1961)
[2] C. Mankoc, A. Janda, R. Arevalo, J. Pastor, I. Zuriguel, A. Garcimartín, D. Maza, Granul. Matter 9, 407 (2007)

[3] A. Janda, I. Zuriguel, D. Maza, Phys Rev. Lett. 108, 248001 (2012)

[4] M. Benyamine, P. Aussillous, B. Dalloz-Dubrujeaud, Discharge flow of a granular media from a silo: effect of the packing fraction and of the hopper angle, in EPJ Web Conf. (EDP Sciences, 2017), Vol. 140, p. 03043

[5] D. Höhner, S. Wirtz, V. Scherer, Powder Technol. 278, 286 (2015)

[6] A. Anand, J.S. Curtis, C.R. Wassgren, B.C. Hancock, W.R. Ketterhagen, Chem. Eng. Sci 64, 5268 (2009)

[7] K. To, P.Y. Lai, H. Pak, Phys. Rev. lett. 86, 71 (2001)

[8] A. Janda, I. Zuriguel, A. Garcimartín, L.A. Pugnaloni, D. Maza, EPL 84, 44002 (2008)

[9] C. Mankoc, A. Garcimartín, I. Zuriguel, D. Maza, L.A. Pugnaloni, Phys. Rev. E 80, 011309 (2009)

[10] I. Zuriguel, Á. Janda, R. Arévalo, D. Maza, Á. Garcimartín, Clogging and unclogging of many-particle systems passing through a bottleneck, in EPJ Web Conf. (EDP Sciences, 2017), Vol. 140, p. 01002

[11] S.K. de Richter, G. Le Caër, R. Delannay, EPL 85, 58004 (2009)

[12] C. Hanotin, P. Marchal, L.J. Michot, C. Baravian, S.K. de Richter, Soft Matter 9, 9352 (2013)

[13] N. Gaudel, S.K. de Richter, N. Louvet, M. Jenny, S. Skali-Lami, Phys. Rev. E 96, 062905 (2017)

[14] A. Pascot, N. Gaudel, S. Antonyuk, J. Bianchin, S.K. De Richter, Chem. Eng. Sci 224, 115749 (2020)

[15] C. Kloss, C. Goniva, A. Hager, S. Amberger, S. Pirker, Prog. Comput. Fluid Dy. 12, 140 (2012)

[16] A. Di Renzo, F.P. Di Maio, Chem. Eng. Sci 60, 1303 (2005)

[17] C.R. Wassgren, M.L. Hunt, P. Freese, J. Palamara, C. Brennen, Phys. Fluids 14, 3439 (2002)

[18] H. Rinne, The Weibull distribution: a handbook (Chapman and Hall/CRC, 2008)

[19] J.M. Pastor, A. Garcimartín, P.A. Gago, J.P. Peralta, C. Martín-Gómez, L.M. Ferrer, D. Maza, D.R. Parisi, L.A. Pugnaloni, I. Zuriguel, Phys. Rev. E 92, 062817 (2015)

[20] A. Goldshtein, M. Shapiro, L. Moldavsky, M. Fichman, J. Fluid Mech. 287, 349 (1995)

[21] J. Bougie, S.J. Moon, J. Swift, H.L. Swinney, Phys. Rev. E 66, 051301 (2002)

[22] B. Andreotti, Y. Forterre, O. Pouliquen, Granular media: between fluid and solid (Cambridge University Press, 2013) 